\title{
Development of 3D method to assess intramuscular spatial distribution of fat infiltration in patients with rotator cuff tear: reliability and concurrent validity
}

Rajan Khanna', Matthew D. Saltzman², James M. Elliott ${ }^{3,4}$, Mark A. Hoggarth ${ }^{4}$, Guido M. Marra², Imran Omar ${ }^{5}$, Todd Parrish ${ }^{5}$ and Amee L. Seitz ${ }^{4^{*}}$ (D)

\begin{abstract}
Background: Intramuscular fat infiltration is a critical factor in surgical decision-making and is the most important factor used to prognosticate surgical repair outcomes in patients with rotator cuff tears. Quantitative 3D assessment of total rotator cuff fat infiltration in patients with rotator cuff tears has been realized. However, a reproducible method to evaluate 3D spatial distribution of rotator cuff intramuscular fat has not been established. The objective of this study was to establish the reproducibility, change detectable beyond error, and concurrent validity of a semi-automated method to evaluate the 3D spatial distribution of fat infiltration and muscle volume in patients with rotator cuff tears.

Methods: Thirteen consecutive patients diagnosed with symptomatic rotator cuff pathology and 3.0 T MRI confirmation at a single center were included. Fat-water imaging was used to quantify 3D intramuscular fat (\%fat) in sagittal oblique sequences and intramuscular spatial distribution with the semi-automated technique. Each rotator cuff muscle was manually segmented yielding \%fat in four axial intramuscular quartile-regions (superior-inferior; Q1-4) and three sagittal (medial/ intermediate/ lateral) regions. Reliability and concurrent validity of \%fat and whole muscle volume were calculated with intraclass correlation coefficients (ICC).

Results: Intra-rater reliability for intramuscular sagittal divisions (ICC $=0.93-0.99)$ and axial divisions (ICC $=0.78-0.99$ ) was good/excellent. Inter-rater reliability for \%fat (ICC $=0.82-0.99)$ and volume $(I C C=0.92-0.99)$ was good/excellent. Concurrent validity with commercialized software showed good/excellent agreement (ICC $=0.66-0.99$ ).

Conclusions: A new semi-automated method to assess 3-dimensional intramuscular distribution of fat infiltration in patients with rotator cuff tears using advanced MR imaging demonstrates high intra and inter-rater reliability and good concurrent validity. Minimal detectable change thresholds established facilitate clinical interpretation for future clinical application of this technique to assess change and treatment efficacy in patients with rotator cuff tears.
\end{abstract}

Keywords: Dixon MRI, shoulder, muscle degeneration, Atrophy, Supraspinatus

\footnotetext{
* Correspondence: amee.seitz@northwestern.edu

${ }^{4}$ Department of Physical Therapy \& Human Movement Sciences, Feinberg

School of Medicine, Northwestern University, Chicago, IL, USA

Full list of author information is available at the end of the article
}

(c) The Author(s). 2019 Open Access This article is distributed under the terms of the Creative Commons Attribution 4.0 International License (http://creativecommons.org/licenses/by/4.0/), which permits unrestricted use, distribution, and reproduction in any medium, provided you give appropriate credit to the original author(s) and the source, provide a link to the Creative Commons license, and indicate if changes were made. The Creative Commons Public Domain Dedication waiver (http://creativecommons.org/publicdomain/zero/1.0/) applies to the data made available in this article, unless otherwise stated. 


\section{Background}

The fat infiltration and atrophy of the rotator cuff muscles are negative predictors of surgical outcomes [1-3] and, therefore, are critical factors to evaluate in the clinical management of patients with a symptomatic rotator cuff (RC) tear. Timely surgical repair of RC tear prior to progressive [4] and potentially irreversible physiological changes in muscle physiology [3] has been advocated, but non-surgical management in the cases of degenerative RC tear is also an effective option. Thus, the ability for clinicians to precisely detect the onset and rate of temporal rotator cuff muscle degeneration may assist with clinical decision making to determine the optimal treatment, timing, and efficacy of surgical repair for the individual patient with RC tear.

Muscle atrophy and fat infiltration are clinically evaluated in a sagittal oblique magnetic resonance image in a few slices at the Y-view (Fig. 1.). Atrophy is assessed clinically with an occupation ratio [5] or tangent line [6] permitting surgeons to visually estimate the muscle size relative to the surrounding bone (scapula). Fat infiltration of the rotator cuff muscles is also visually appreciated and rated in the clinic with clinic-friendly qualitative scales (i.e. Goutallier or Fuchs) [7, 8]. Despite the reported predictive value for these evaluative radiological scales to evaluate intramuscular fat, reproducibility and accuracy have been challenged $[9,10]$. Additionally, the precision of these clinical scales do not allow for assessment of temporal changes that occur before significant moderate to severe degeneration negatively impacts the reparability and patient outcome [1-3]. For these reasons, more quantitative methods are necessary.

Advanced MRI techniques that allow for greater precision and accuracy towards quantifying intramuscular fat by using chemical shift-based water-fat techniques, such as the Dixon [11] or IDEAL methods are more readily available. With these methods, data are collected at an echo time when water and fat are in-phase and at an echo time when the phases are opposed. The data can then be combined to generate a fat and water image allowing for calculation of percent fat infiltration (\%fat). These quantitative techniques are not new and have been used to evaluate the magnitude of muscle \%fat in individuals with a variety of musculoskeletal disorders [12-15] and have demonstrated concurrent validity with positive correlation to clinical grading of fat, [16] tear size, [17] and biopsy results [18].

Typically quantitative techniques have assessed \%fat in a single slice or several slices near the scapular $y$-view have demonstrated construct validity with associations to the clinical Goutallier grading of muscle [16] and the size of the tear [17]. However, the accuracy of a 2D assessment in a single or few $y$-view slice images has also been challenged in the presence of tendon retraction or with inhomogeneous degenerative changes. Recently, Matsumura, et al. [19] established the reliability of 3D assessment for intramuscular fat\% taking the average of all slices across the entire muscle in patients with rotator cuff tears. While such work clearly advances our understanding of total muscle composition, the technique does not capture or appreciate the heterogeneity of intramuscular fat infiltration in rotator cuff tears [20]. The ability to appreciate inhomogeneous distribution of intramuscular fat within the rotator cuff muscles will facilitate further understanding of the temporal progression of muscle degeneration with rotator cuff tears. Yet, there are currently no methods to evaluate spatial distribution of intramuscular \%fat of the rotator cuff with established reliability or validity.

Thus, we seek to expand on the existing 3D imaging methods used to quantify physiological degenerative changes in rotator cuff muscles by first, establishing measurement properties using sagittal oblique slices, and second to report on the feasibility and reliability of a novel semi-automated method to assess the 3D intramuscular fat distribution to allow for detection of
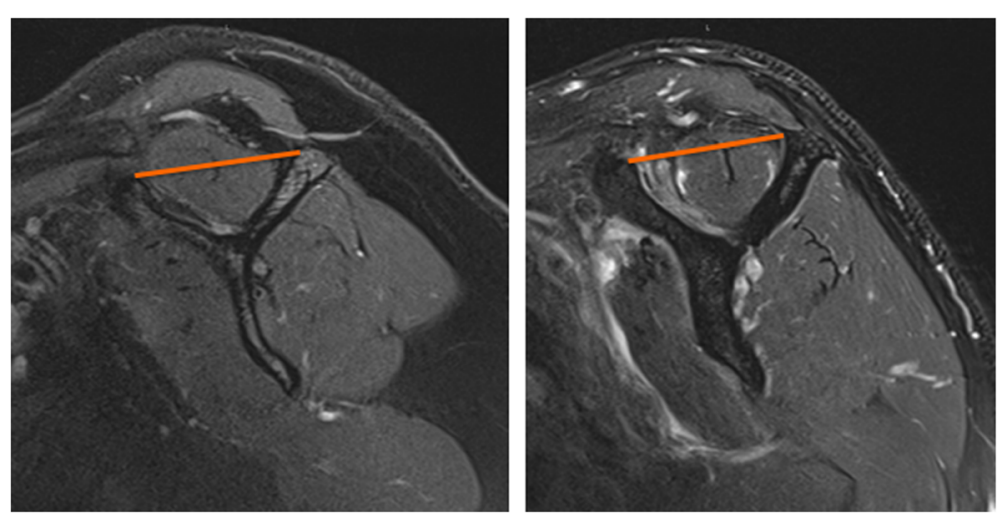

Fig. 1 The Y-view used to clinically grade rotator cuff muscle fat infiltration and atrophy. Normal supraspinatus muscle (Left) and degeneration of the supraspinatus with atrophy and fat infiltration (Right). a tangent line (Orange) is used to evaluate the amount of atrophy 
inhomogeneous changes. The purpose of this study is to establish the reproducibility, change detectable beyond measurement error (MDC), and concurrent validity to define $3 \mathrm{D}$ whole muscle volume and spatial distribution of intramuscular fat\% in both the axial and sagittal planes in patients with rotator cuff tears.

\section{Methods}

\section{Subjects}

This study was a single-center study of patients seen by one of two fellowship trained shoulder surgeons between December 2015 and May 2016. Consecutive patients diagnosed with rotator cuff tendon pathology and confirmed with MR imaging at this institution were retrospectively identified and included in the study. This subset of patients was selected to maintain consistency in the scanner used for MR imaging and sequences available for analysis. The surgeon diagnosis of rotator cuff tendon pathology included a positive finding in at least 3 of the following clinical tests: Hawkins Test, Neer Sign, Painful Arc, External Rotation Lag Sign, Hornblower Sign, Pain with External Rotation Resistance or a Jobe Sign. Exclusion criteria included a limitation in passive shoulder range of motion in 3 or more planes consistent with adhesive capsulitis. Patients with moderate to severe glenohumeral joint osteoarthritis, prior rotator cuff repair or other shoulder surgery, and patients with history of a shoulder fracture or deformity from prior fractures were also excluded. A fellowship trained, board certified musculoskeletal radiologist with over 12 years of experience, independently read the MR images blinded to the surgeon diagnosis. Patients determined to have both symptomatic clinical diagnosis and findings on MRI of rotator cuff pathology were included. Rotator cuff tendon pathology was classified by the radiologist as no tear, partial thickness tear, and full-thickness tear small $<1 \mathrm{~cm}$, medium $1-3 \mathrm{~cm}$, large $>3-5 \mathrm{~cm}$, or massive $>5 \mathrm{~cm}$. The radiologist also qualitatively graded fat infiltration using the Goutallier Classification. Using standardized methods, [21] the sample size estimate was 10 subjects using a one-sided $\alpha=0.05$, and $\beta=0.80$ to yield $80 \%$ power to detect an acceptable reliability Intraclass Correlation Coefficient (ICC) of 0.75 and an expected ICC of 0.95 based on previous research [19]. We, therefore, included the first 13 patients meeting inclusion/exclusion criteria in this study. The study was approved by the Institutional Review Board and conducted in accordance with the Committee for Human Research.

\section{Shoulder MRI protocol}

Magnetic resonance images were acquired according to service institutional standards using a 3-T Siemens (Skyra, Siemens, Erlangen, Germany) magnetic resonance scanner with a 16-channel phased array shoulder coil. To ensure coverage of the rotator cuff muscles, the sagittal oblique MR imaging sequence with respect to the glenoid fossa was planned on an axial scout scan with a field of view of $18 \times 18 \mathrm{~cm}$, which was fit to include the medial border of the scapula. T1-weighted sequences which include fast spin-echo sequences in oblique coronal and sagittal planes, a multiple echo data image continuation (MEDIC) sequence in the axial plane and a T2\%-weighted fat suppressed sequences in the axial plane, coronal oblique, and sagittal oblique planes were also performed. Finally, a 3D multi-echo two-point Dixon fat/water imaging sequence was performed in the sagittal oblique plane. This sequence is a chemical-shift imaging application producing water- and fat-only images from dual echo acquisitions with precise accuracy for \%fat quantification [18, 22, 23]. The imaging parameters were as follows: slice thickness $2.0 \mathrm{~mm}$, TR/TE1/ TE2 $=3.97 \mathrm{~ms} / 1.29 \mathrm{~ms} / 2.52 \mathrm{~ms}$, Flip angle $9^{\circ}, 380 \mathrm{~mm}$ FOV, and acquisition matrix of $320 \times 320$ with 120 slices to produce a voxel resolution of $1.2 \times 1.2 \times 2.0 \mathrm{~mm}$ and $1040 \mathrm{~Hz} / \mathrm{Px}$ bandwidth. In order to reduce aliasing in both the phase and 3D direction, oversampling of 100 and $60 \%$ were used respectively. To reduce the acquisition time, an acceleration factor of 2 was used in both the slice and phase directions.

\section{Image analysis}

The 3D intramuscular \%fat and muscle volume were quantified by manual segmentation of rotator cuff muscles on the Dixon fat-water sequences using custom software in Matlab (Mathworks V, Natick MA). Two examiners without experience reading MR images were trained in two 30-min sessions by a musculoskeletal radiologist and 2 orthopedic surgeons to identify the boarders of the regions of interest. To establish inter-rater reliability, both examiners independently performed segmentation of the regions of interest on fat-water sequence MR images, blinded to subject identifiers, tear size, and each other's results. To establish feasibility and intra-rater test-retest reliability, one examiner repeated the segmentation 6-weeks later also blind to subject identifiers; tear size, and prior segmentation results. Lastly, we examined concurrent validity of \%fat and muscle volumes with manual segmentation of the regions of interest using commercially available software (AnalyzeDirect Software, V. 11.0) with the same fat-water imaging processing parameters. This system has been used to assess intramuscular fat [12-15] and previously validated with the gold-standard, biopsy [18].

All segmentation was performed manually inside the fascial borders the defined the regions of interest in supraspinatus (SS), combined infraspinatus/teres minor (IS), subscapularis (SC) muscles in each oblique sagittal image (Fig. 2.). The MATLAB program generated \%fat 


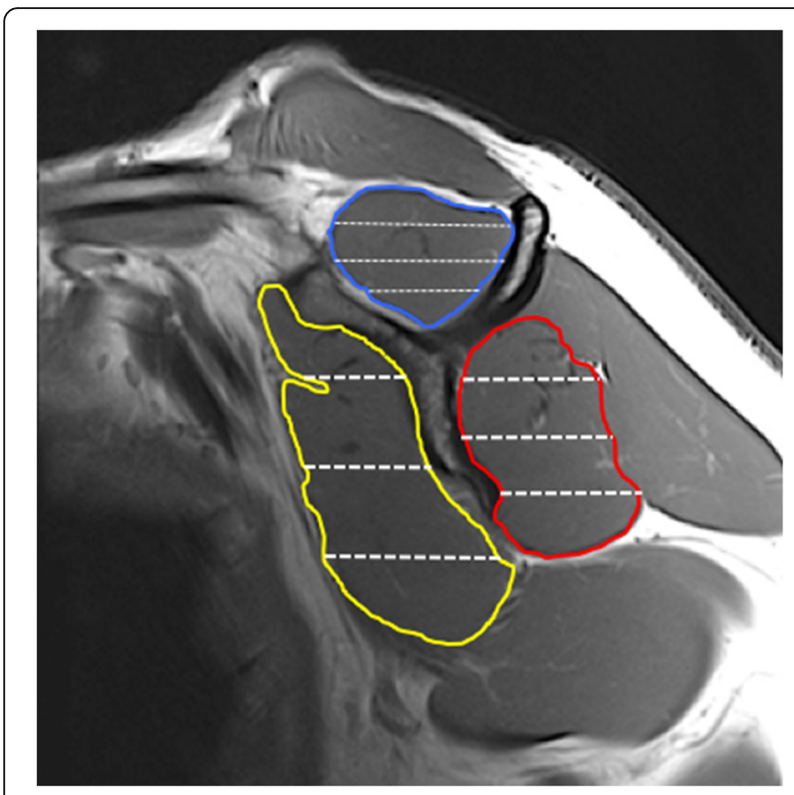

Fig. 2 Manual segmentation of the supraspinatus (Blue), combined infraspinatus/teres minor (Red), subscapularis (Yellow). Spatial distribution of intramuscular \%fat in automated superior to inferior quartiles was derived for each muscle

data for quartilesin each of the segmented muscles to evaluate spatial distribution of \%fat by quartile superiorly to inferiorly (Q1-Q4) (Fig. 2.). Consistent with methods described by prior investigators, the infraspinatus and teres minor muscles were segmented and evaluated as one $[6,24,25]$. Using these muscular regions of interest, two sets of co-registered fat-water images were used to calculate fat fraction (\%fat) with the following equation:

Fat Fraction $=$ Fat $/($ Fat + Water $) \times 100$.

Sequential images from the most lateral image at the level of the glenoid to the most medial image of the medial scapular boarder were processed. Thus, the number of images used in the oblique sagittal view varied by subject, due to individual subject size differences needed to capture the scapula and corresponding rotator cuff muscles. The average of all consecutive images was used to establish the 3D total muscle \%fat of each muscle. The $3 \mathrm{D}$ volume of each muscle was calculated by using the sum of the area calculated in each slice taking into account slice thickness from the glenoid to the medial boarder of the scapula. In addition to 3D whole muscle measures, three sagittal intra-muscular tertiles (lateral, intermediate, and medial) were created by dividing each muscle from the glenoid to the medial boarder of the scapula into equal thirds (Fig. 3) whereby average \%fat and volume of each intra-muscular region were calculated.

\section{Statistical analysis}

The relative intra and inter-rater reliability of 3D \%fat and volume of the entire muscle and intramuscular divisions were determined by calculating the Intraclass Correlation Coefficient (ICC) [26] and 95\% Confidence Intervals $(95 \% \mathrm{CI})$. For all analyses, the ICC values were considered: poor if below 0.20; fair from 0.21 to 0.40 ; moderate from 0.41 to 0.60 ; good from 0.61 to 0.80 ; and very good from 0.81 to 1.00 [27]. The absolute reliability was defined as the standard error of the measure (SEM), representing error associated with a single measure [28], and the minimal detectable change (MDC), representing the value that exceeds error associated with repeated measures [29]. The MDC is used to interpret changes in fat infiltration and volume that are necessary to exceed measurement error. The concurrent validity was also determined with ICCs by comparing values between those derived with current methods and previously validated commercial software. All statistical analyses were conducted with the IBM SPSS Statistics software (Version 23; IBM Corp, Armonk, NY).

\section{Results}

Intra and inter-rater reliability was assessed in MR imaging of 13 consecutive patients (5 males, 8 females) who met the inclusion criteria. These patients had a mean age of $51.0 \pm 16.5( \pm \mathrm{SD})$ years with 7 patients with partial-thickness tears, 2 patients with small full-thickness tears, and 4 patients with medium -sized full-thickness tears. Goutallier grade 2 fat infiltration was identified in the supraspinatus in one patient, and grade 1 fat infiltration in 4 patients. The infraspinatus showed grade 1 fat infiltration in 4 patients, and the teres minor showed grade 1 fat infiltration in one patient. The mean chronicity of patient's symptoms was $38.5 \pm 78.0$ months. The intra-rater absolute reliability of spatial distribution methods evaluating 3D fat infiltration and 3D muscle volume of the rotator cuff muscles was considered very good to excellent from medial to lateral tertiles (Table 1) and \%fat (Table 2) from superior to inferior quartiles (Q1-Q4). Relative reliability, with the SEM and MDC expressed in units of \%fat, volume was also established and shown. The inter-rater absolute reliability assessing the spatial distribution of 3D fat infiltration and volume of the rotator cuff muscles was considered good to excellent from medial to lateral tertiles (Table 3) and \%fat (Table 4) from superior to inferior quartiles (Q1-Q4). With regard to current validity (Table 5), the ICCs for agreement between the two methods was also very good to excellent for both fat\% and volume across the entire muscle.

\section{Discussion}

Results of this study show the feasibility of advanced MR imaging techniques using a novel semi-automated method to quantify the spatial distribution of 3D rotator cuff muscle fat infiltration in patients with rotator 


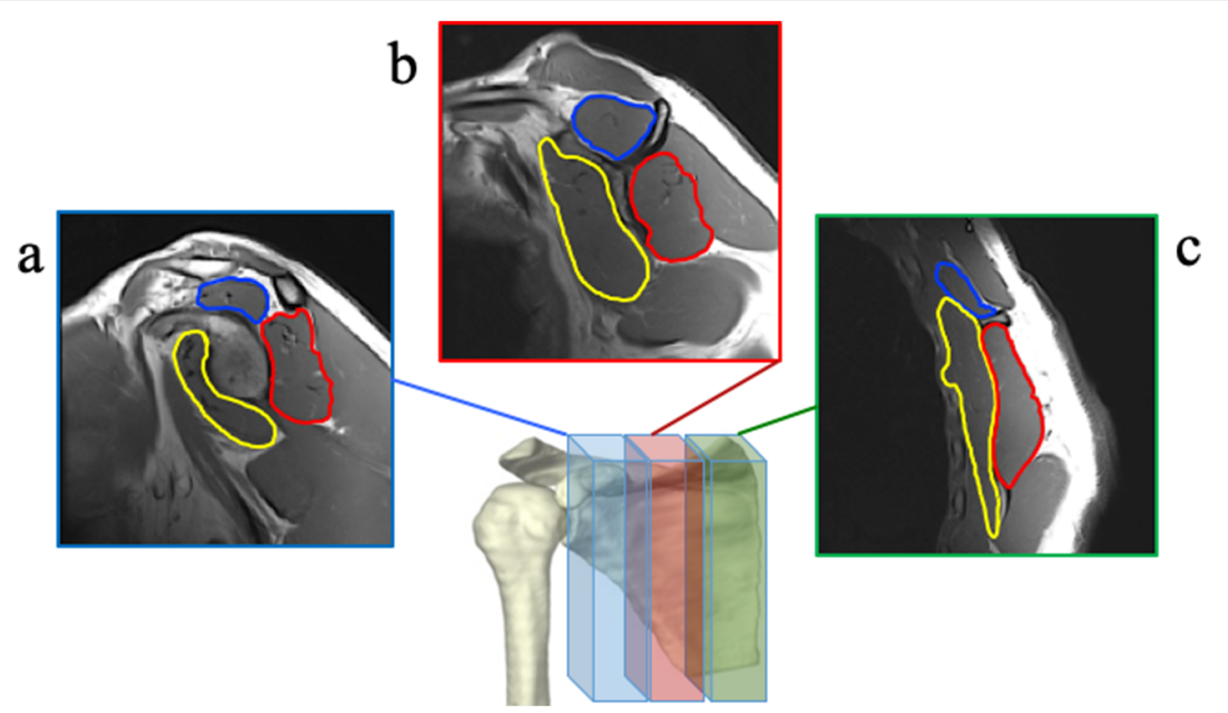

Fig. 3 Reliability of the spatial distribution of \%fat was calculated to derive \%fat in the: a) lateral, b) intermediate and c) medial intramuscular tertile for each rotator cuff muscle. The mean total muscle \%fat and volume were calculated from all regions

Table 1 Intra-rater reliability of 3D \%fat in superior to inferior quartile $(\mathrm{Q} 1-\mathrm{Q} 4)$ regions in patients $(N=13)$ with rotator cuff pathology

\begin{tabular}{|c|c|c|c|c|c|}
\hline \multicolumn{6}{|l|}{ Intra-rater Reliability } \\
\hline \multirow{3}{*}{$\begin{array}{l}\text { Rotator Cuff Muscle } \\
\text { Intramuscular region }\end{array}$} & \multicolumn{5}{|c|}{ 3D \%Fat } \\
\hline & \multicolumn{2}{|c|}{ Absolute } & \multicolumn{3}{|c|}{ Relative } \\
\hline & ICC & $95 \% \mathrm{Cl}$ & Mean & SEM & MDC \\
\hline \multicolumn{6}{|l|}{ Supraspinatus (whole) } \\
\hline Q1 & 0.960 & $0.872,0.988$ & 13.85 & 1.74 & 2.46 \\
\hline Q2 & 0.991 & $0.971,0.997$ & 12.85 & 0.73 & 1.04 \\
\hline Q3 & 0.988 & $0.961,0.996$ & 12.43 & 0.85 & 1.20 \\
\hline Q4 & 0.927 & $0.770,0.977$ & 14.19 & 2.38 & 3.36 \\
\hline \multicolumn{6}{|l|}{ Infra/Teres (whole) } \\
\hline Q1 & 0.993 & $0.977,0.998$ & 10.38 & 0.55 & 0.78 \\
\hline Q2 & 0.994 & $0.981,0.998$ & 11.42 & 0.61 & 0.86 \\
\hline Q3 & 0.984 & $0.948,0.995$ & 12.50 & 0.96 & 1.36 \\
\hline Q4 & 0.945 & $0.821,0.983$ & 10.96 & 1.54 & 2.18 \\
\hline \multicolumn{6}{|l|}{ Subscapularis (whole) } \\
\hline Q1 & 0.782 & $0.280,0.934$ & 13.04 & 2.95 & 4.17 \\
\hline Q2 & 0.996 & $0.987,0.999$ & 13.77 & 0.54 & 0.77 \\
\hline Q3 & 0.998 & $0.994,0.999$ & 13.77 & 0.44 & 0.64 \\
\hline Q4 & 0.995 & $0.963,0.999$ & 15.35 & 0.75 & 1.06 \\
\hline
\end{tabular}

ICC intraclass correlation coefficient, $95 \% \mathrm{Cl}, 95 \%$ confidence interval, SEM standard error of the measure, MDC minimal detectable change cuff pathology. Furthermore, these methods demonstrate good to excellent reliability and concurrent validity with previously validated fat fraction and volumetric methods [18].

In prior studies using quantitative $2 \mathrm{D}$ methods, reliability of \%fat with intraclass correlation coefficients (ICC) of 0.60 for the supraspinatus, 0.65 of the infraspinatus using 4 consecutive lateral slices adjacent to the sagittal oblique "scapular y-view" were reported [16]. Another study reported higher test-retest reliability of $\%$ fat in the rotator cuff ranging from ICC $=0.895$ to 0.952 [30]. Similarly, volume estimates of the entire muscle have been performed using selected cross-section area measures of the muscle [31] and cross-sectional area based atrophy ratios [6]. However, recent evidence suggests there is variation in \%fat regionally within the rotator cuff muscles in individuals with rotator cuff pathology [20]. Thus 2D measures using a single slice or several consecutive slices adjacent to the $y$-view may not provide the most sensitive test towards detecting the disparate spatial distribution and magnitude of temporal changes in fat infiltration.

To adequately evaluate temporal changes of fat and atrophy in rotator cuff muscles in patients with tendon pathology, 3D quantification could appreciate early inhomogeneous degeneration following a rotator cuff tear before irreversible change occurs. Matsumura et al., recently demonstrated excellent reliability with 3D \%fat and volume using a standardized protocol $(3 \mathrm{~T}, 1 \mathrm{~mm}$ slice) in axial slices [19]. Our results show comparable 3D whole muscle \%fat and volume data. Furthermore, the technical advances in 3D methods in the current study 
Table 2 Intra-rater reliability of 3D \%fat and volume in medial to lateral (tertile) regions patients $(N=13)$ with rotator cuff pathology

\begin{tabular}{|c|c|c|c|c|c|c|c|c|c|c|}
\hline \multirow{4}{*}{$\begin{array}{l}\text { Rotator Cuff Muscle } \\
\text { Intramuscular region }\end{array}$} & \multicolumn{10}{|c|}{ Intra-rater Reliability } \\
\hline & \multicolumn{5}{|c|}{ 3D \%Fat } & \multicolumn{5}{|c|}{ 3D Volume $(\mathrm{cm} 3)$} \\
\hline & \multicolumn{2}{|c|}{ Absolute } & \multicolumn{3}{|c|}{ Relative } & \multicolumn{2}{|c|}{ Absolute } & \multicolumn{3}{|c|}{ Relative } \\
\hline & ICC & $95 \% \mathrm{Cl}$ & Mean & SEM & $\mathrm{MDC}$ & ICC & $95 \% \mathrm{Cl}$ & Mean & SEM & MDC \\
\hline Supraspinatus (whole) & 0.974 & $0.915,0.992$ & 11.69 & 1.10 & 1.56 & 0.958 & $0.869,0.987$ & 29.6 & 2.9 & 4.1 \\
\hline Lateral Region & 0.935 & $0.794,0.980$ & 10.38 & 1.20 & 1.70 & 0.689 & $-0.036,0.906$ & 8.1 & 1.9 & 2.7 \\
\hline Intermediate Region & 0.980 & $0.936,0.994$ & 11.65 & 1.08 & 1.53 & 0.967 & $0.890,0.990$ & 11.4 & 1.0 & 1.4 \\
\hline Medial Region & 0.975 & $0.918,0.992$ & 13.03 & 1.49 & 2.11 & 0.996 & $0.988,0.999$ & 10.1 & 0.4 & 0.6 \\
\hline Infra/Teres (whole) & 0.989 & $0.966,0.997$ & 10.51 & 0.68 & 0.96 & 0.979 & $0.929,0.994$ & 65.7 & 3.5 & 5.0 \\
\hline Lateral Region & 0.968 & $0.897,0.990$ & 10.54 & 1.12 & 1.58 & 0.935 & $0.789,0.980$ & 19.1 & 1.7 & 2.4 \\
\hline Intermediate Region & 0.977 & $0.927,0.993$ & 10.76 & 1.00 & 1.42 & 0.976 & $0.924,0.993$ & 23.0 & 1.2 & 1.8 \\
\hline Medial Region & 0.957 & $0.865,0.987$ & 10.22 & 1.41 & 1.99 & 0.978 & $0.920,0.993$ & 23.6 & 1.8 & 2.5 \\
\hline Subscapularis (whole) & 0.996 & $0.986,0.999$ & 13.12 & 0.47 & 0.67 & 0.910 & $0.701,0.973$ & 78.2 & 8.1 & 11.4 \\
\hline Lateral Region & 0.984 & $0.945,0.995$ & 13.73 & 0.97 & 1.38 & 0.879 & $0.595,0.963$ & 20.5 & 3.2 & 4.6 \\
\hline Intermediate Region & 0.996 & $0.987,0.999$ & 13.14 & 0.48 & 0.68 & 0.983 & $0.945,0.995$ & 31.6 & 1.5 & 2.1 \\
\hline Medial Region & 0.995 & $0.984,0.999$ & 13.04 & 0.56 & 0.79 & 0.956 & $0.797,0.988$ & 26.3 & 2.8 & 3.9 \\
\hline
\end{tabular}

ICC intraclass correlation coefficient, $95 \% \mathrm{Cl}, 95 \%$ confidence interval, SEM standard error of the measure, MDC minimal detectable change

provide feasibility and reliability of a semi-automated technique to quantify intramuscular distribution mediallateral (tertiles) and superior-inferior (quartiles) to facilitate quantification of inhomogeneous 3D fat infiltration. Since muscles are 3D structures, imaging methods that best capture 3D rotator cuff muscle fat and atrophic changes of the in patients with rotator cuff tears [19]. Spatial distribution of \%fat using the Dixon sequence in cervical and lumbar spine musculature has been established [13, 22, 32]. However, to our knowledge the reliability of evaluating the spatial distribution of \% fat of rotator cuff muscles has not been previously reported, which supports the novelty of the processing methods. Additionally, the current study demonstrates excellent reliability and concurrent validity of 3D whole muscle and intramuscular regional distribution assessment of \%fat and volume with commercial software, providing additional support of the validity of our methods.

In addition to establishing absolute reliability, we also present error threshold data relevant to interpreting meaningfulness of statistical results for fat infiltration and volume measures. The SEM and MDC are the

Table 3 Inter-tester reliability of 3D \%fat and volume for whole muscle and medial to lateral (tertile) regions in patients $(N=13)$ with rotator cuff pathology

\begin{tabular}{|c|c|c|c|c|c|c|c|c|c|c|}
\hline \multirow{4}{*}{$\begin{array}{l}\text { Rotator Cuff Muscle } \\
\text { Intramuscular region }\end{array}$} & \multicolumn{10}{|c|}{ Inter-rater Reliability } \\
\hline & \multicolumn{5}{|c|}{ 3D \%Fat } & \multicolumn{5}{|c|}{ 3D Volume } \\
\hline & \multicolumn{2}{|c|}{ Absolute } & \multicolumn{3}{|c|}{ Relative (\%fat) } & \multicolumn{2}{|c|}{ Absolute } & \multicolumn{3}{|c|}{ Relative (cm3) } \\
\hline & $\mathrm{ICC}$ & $95 \% \mathrm{Cl}$ & Mean & SEM & $\mathrm{MDC}$ & $\mathrm{ICC}$ & $95 \% \mathrm{Cl}$ & Mean & SEM & MDC \\
\hline Supraspinatus (whole) & 0.979 & $0.861,0.995$ & 11.03 & 0.87 & 1.22 & 0.971 & $0.910,0.991$ & 29.9 & 2.5 & 3.5 \\
\hline Lateral Region & 0.820 & $0.218,0.950$ & 8.88 & 1.73 & 2.45 & 0.735 & $0.160,0.918$ & 7.9 & 1.9 & 2.7 \\
\hline Intermediate Region & 0.984 & $0.930,0.996$ & 11.26 & 0.89 & 1.25 & 0.982 & $0.940,0.994$ & 11.8 & 0.7 & 1.0 \\
\hline Medial Region & 0.948 & $0.829,0.984$ & 12.80 & 1.81 & 2.56 & 0.989 & $0.966,0.997$ & 10.1 & 0.7 & 0.9 \\
\hline Infra/Teres (whole) & 0.992 & $0.947,0.998$ & 10.05 & 0.55 & 0.78 & 0.973 & $0.897,0.992$ & 69.3 & 4.2 & 6.0 \\
\hline Lateral Region & 0.990 & $0.968,0.997$ & 10.50 & 0.65 & 0.91 & 0.923 & $0.745,0.977$ & 19.5 & 2.0 & 2.8 \\
\hline Intermediate Region & 0.990 & $0.931,0.998$ & 10.19 & 0.64 & 0.90 & 0.966 & $0.824,0.991$ & 24.3 & 1.5 & 2.2 \\
\hline Medial Region & 0.984 & $0.940,0.995$ & 9.53 & 0.76 & 1.07 & 0.944 & $0.820,0.983$ & 25.5 & 2.7 & 3.8 \\
\hline Subscapularis (whole) & 0.996 & $0.989,0.999$ & 12.75 & 0.47 & 0.66 & 0.955 & $0.857,0.986$ & 83.1 & 6.2 & 8.8 \\
\hline Lateral Region & 0.989 & $0.964,0.997$ & 13.34 & 0.79 & 1.12 & 0.629 & $0.369,0.889$ & 20.1 & 4.5 & 6.4 \\
\hline Intermediate Region & 0.990 & $0.952,0.997$ & 12.60 & 0.77 & 1.09 & 0.962 & $0.874,0.988$ & 32.9 & 2.4 & 3.4 \\
\hline Medial Region & 0.996 & $0.987,0.999$ & 12.20 & 0.51 & 0.72 & 0.840 & $0.473,0.951$ & 30.1 & 4.9 & 6.9 \\
\hline
\end{tabular}

ICC intraclass correlation coefficient, $95 \% \mathrm{Cl}, 95 \%$ confidence interval, SEM standard error of the measure, MDC minimal detectable change 
Table 4 Inter-rater reliability of 3D \%fat in superior to inferior quartiles (Q1-Q4) in patients ( $N=13)$ with rotator cuff pathology

\begin{tabular}{|c|c|c|c|c|c|}
\hline \multirow{4}{*}{$\begin{array}{l}\text { Rotator Cuff Muscle } \\
\text { Intramuscular region }\end{array}$} & \multicolumn{5}{|c|}{$\underline{\text { Inter-rater Reliability }}$} \\
\hline & \multicolumn{5}{|c|}{ 3D \%Fat } \\
\hline & \multicolumn{2}{|c|}{ Absolute } & \multicolumn{3}{|c|}{ Relative } \\
\hline & $\mathrm{ICC}$ & $95 \% \mathrm{Cl}$ & Mean & SEM & MDC \\
\hline \multicolumn{6}{|l|}{ Supraspinatus (whole) } \\
\hline Q1 & 0.980 & $0.936,0.994$ & 13.26 & 1.09 & 1.54 \\
\hline Q2 & 0.978 & $0.879,0.994$ & 12.27 & 1.05 & 1.48 \\
\hline Q3 & 0.982 & $0.881,0.995$ & 12.12 & 0.98 & 1.39 \\
\hline Q4 & 0.926 & $0.300,0.983$ & 12.03 & 1.73 & 2.45 \\
\hline \multicolumn{6}{|l|}{ Infra/Teres (whole) } \\
\hline Q1 & 0.996 & $0.986,0.999$ & 10.09 & 0.40 & 0.57 \\
\hline Q2 & 0.997 & $0.989,0.999$ & 11.18 & 0.42 & 0.59 \\
\hline Q3 & 0.984 & $0.946,0.995$ & 11.85 & 0.90 & 1.28 \\
\hline Q4 & 0.963 & $0.469,0.992$ & 9.87 & 1.13 & 1.60 \\
\hline \multicolumn{6}{|l|}{ Subscapularis (whole) } \\
\hline Q1 & 0.991 & $0.971,0.997$ & 11.84 & 0.51 & 0.72 \\
\hline Q2 & 0.996 & $0.983,0.999$ & 13.25 & 0.54 & 0.76 \\
\hline Q3 & 0.996 & $0.986,0.999$ & 13.38 & 0.61 & 0.86 \\
\hline Q4 & 0.997 & $0.991,0.999$ & 14.89 & 0.58 & 0.82 \\
\hline
\end{tabular}

ICC intraclass correlation coefficient, $95 \% \mathrm{Cl} 95 \%$ confidence interval, SEM standard error of the measure; MDC, minimal detectable change

errors associated with a single measurement and repeated measures, respectively, in the units of measure (\%fat and $\mathrm{cm}^{3}$ volume). The intra-rater MDC was less than $2 \%$ for each rotator cuff muscle and less than $2.2 \%$ for the intramuscular spatial distribution measures of

Table 5 Concurrent validity of 3D total and medial to lateral region \%fat and volume

\begin{tabular}{|c|c|c|c|c|}
\hline \multirow{3}{*}{$\begin{array}{l}\text { Rotator Cuff Muscle } \\
\text { Intramuscular region }\end{array}$} & \multicolumn{4}{|c|}{ Validity } \\
\hline & \multicolumn{2}{|c|}{ 3D \%Fat } & \multicolumn{2}{|c|}{ Volume } \\
\hline & $\overline{I C C}$ & $95 \% \mathrm{Cl}$ & $\overline{I C C}$ & $95 \% \mathrm{Cl}$ \\
\hline Supraspinatus (whole) & 0.859 & $0.015,0.970$ & 0.939 & $0.276,0.987$ \\
\hline Lateral Region & 0.655 & $0.250,0.907$ & 0.778 & $0.070,0.942$ \\
\hline Intermediate Region & 0.844 & $0.083,0.963$ & 0.867 & $0.153,0.968$ \\
\hline Medial Region & 0.947 & $0.830,0.984$ & 0.884 & $0.675,0.963$ \\
\hline Infra/Teres (whole) & 0.989 & $0.965,0.997$ & 0.969 & $0.889,0.991$ \\
\hline Lateral Region & 0.969 & $0.905,0.991$ & 0.882 & $0.384,0.969$ \\
\hline Intermediate $\mathrm{F}$ & 0.976 & 0 & 0.937 & 0.78 \\
\hline Medial Region & 0.980 & $0.968,0.997$ & 0.911 & $0.743,0.972$ \\
\hline is (whole) & 0.975 & 0.87 & 0.912 & $0.313,0.979$ \\
\hline Lateral Region & 0.951 & $0.852,0.985$ & 0.580 & $0.037,0.854$ \\
\hline Intermediate Region & 0.978 & $0.907,0.994$ & 0.927 & $0.192,0.985$ \\
\hline Medial Region & 0.949 & $0.694,0.987$ & 0.885 & $0.615,0.965$ \\
\hline
\end{tabular}

ICC intraclass correlation coefficient, $95 \% \mathrm{Cl} 95 \%$ confidence interval, SEM standard error of the measure, $M D C$ minimal detectable change \%fat within the lateral, intermediate, and medial regions of each muscle using our method (Table 1). However, the error of measurement established by the MDC in the superior-inferior quartiles were higher (Table 2) for all muscles $(<2.5 \%)$ with the exception of Q1- the most superior aspect of the subscapularis $(\mathrm{MDC}=4.17 \%)$. The inter-rater MDC (Tables 3 and 4) was less than 1\% for each rotator cuff muscle and intramuscular spatial distribution errors were all less than $2.6 \%$ in both the superior-inferior (quartiles) and medial to lateral (tertiles). This error threshold is important to assist interpretation of emerging research evaluating the efficacy of a healed surgical tendon repair to negate or potentially reverse rotator cuff muscle \%fat in patients with rotator cuff tear [30, 33].

There are several limitations to the study. Overall, the generalizability may be limited as the results were obtained using a single scanner and set of imaging parameters. Additionally, we only examined the intra-rater reliability in one rater and both raters did not have MR image reading experience. While the study exceeded our sample size estimate to provide $>80 \%$ power, sample was small $(n=13)$. However, our results are consistent with the precision previously established, and the time intensive process of manually segmenting each muscle in its entirety limits further efforts for this purpose. Furthermore, we did not include patients with large and massive rotator cuff tears as early detection of temporal change in muscle degeneration is most imperative in patients with less severe rotator cuff disease, such as partial thickness and small-medium full-thickness tears. In these tears, operative treatment is advocated before potentially irreversible changes in the rotator cuff muscle occurs. Thus the population we studied were those who may potentially benefit the most from assessment of temporal physiological muscle changes pre-operatively. Additionally, we combined and segmented the infraspinatus and teres minor together based on previous literature [6] and the difficulty visualizing the fascia plane in sagittal Y-views. However, with the evolution of improved imaging technologies, it is anticipated that the reliable differentiation between these two muscles in sagittal oblique images will be realized and this work is currently underway. Lastly, the pattern of fat infiltration in patients with rotator cuff tears may provide further insight into early temporal changes in muscle degeneration in patients with rotator cuff tears.

\section{Conclusions}

3D MR imaging techniques including, the multi-echo Dixon fat-water sequences allow for reliable and intraand inter-rater quantification of the intramuscular spatial distribution of fat infiltration in patients with rotator cuff pathology. It is suggested that both atrophy 
and fat infiltration should be evaluated separately, given these are two different physiological processes [3, 34, 35]. To adequately evaluate temporal changes of fat and atrophy in rotator cuff muscles in patients with tendon pathology, 3D quantification may be necessary to determine the rate and distribution of muscle degeneration following a rotator cuff tear. While conservative treatment may be advocated for patients with a degenerative rotator cuff tear, determining the rate of muscle degeneration would aid in surgical repair timing decisions. Current study results suggest this method to quantify 3D spatial distribution of muscle pathophysiology is feasible and has utility to reliability quantify rotator cuff muscle degeneration. As future studies utilize more precise 3D measures of muscle atrophy and fat infiltration, the ability to determine the meaningfulness of whether statistical differences exceed measurement error is important; thus the absolute error in units of \% fat was established. The clinical application of this method to evaluate the spatial distribution muscle degeneration may be useful to facilitate surgical and non-surgical treatment choices in patients with rotator cuff tears.

\section{Abbreviations}

\%fat: percent fat infiltration; 3D: Three-dimensional; 95\%Cl: 95\% Confidence Interval; ICC: Intraclass Correlation Coefficient; MDC: minimal detectable change; RC: Rotator Cuff; SEM: Standard error of the measure

\section{Acknowledgements \\ Authors would like to acknowledge Paul Micevych for contributions to data processing.}

\section{Funding}

ALS was funded for this work by a New Investigator Fellowship Training Initiative from the Foundation for Physical Therapy. The funding body had no role in the design of the study, collection, analysis, and interpretation of data and in writing the manuscript.

\section{Availability of data and materials}

Data associated with this study are available in the Feinberg School of Medicine, at Northwestern University. The datasets used and/or analyzed during the current study are not publicly available due to maintenance of privacy but can be made available from the corresponding author upon reasonable request and with institutional permission.

\begin{abstract}
Authors' contributions
ALS conceived, designed, and led the study; MS, GM, TP and IO were responsible for data acquisition for muscle analysis and interpretation of reliability data. ALS and RK performed the data processing, analysis and interpretation. JE and $\mathrm{MH}$ were responsible for data processing and analysis methods. All authors have: made substantial contributions to conception and design, or acquisition of data, or analysis and interpretation of data; been involved in drafting the manuscript or revising it critically for important intellectual content; given final approval of the version to be published; and agreed to be accountable for all aspects of the work in ensuring that questions related to the accuracy or integrity of any part of the work are appropriately investigated and resolved.
\end{abstract}

\section{Ethics approval and consent to participate}

This study received ethics approval from the Northwestern University Institutional Review Board (\#STU00202144) with a waiver of informed written consent as data were retrospectively retrieved, de-identified, and anonymized.

\section{Consent for publication}

NA

\section{Competing interests}

Authors RK, IO, MH, TP and ALS declare that they have no conflicts of interest. MS and GM provide consultation and receive royalties that is not related to this work. JE is principal investigator of an NIH research grant (R01) for investigation related to cervical spine related to whiplash trauma.

\section{Publisher's Note}

Springer Nature remains neutral with regard to jurisdictional claims in published maps and institutional affiliations.

\section{Author details}

${ }^{1}$ Stritch School of Medicine, Loyola University, Chicago, IL, USA. ${ }^{2}$ Department of Orthopedic Surgery, Feinberg School of Medicine, Northwestern University, Chicago, IL, USA. ${ }^{3}$ Northern Sydney Local Health District, The Kolling Research Institute \& Faculty of Health Sciences, University of Sydney, Sydney, Australia. ${ }^{4}$ Department of Physical Therapy \& Human Movement Sciences, Feinberg School of Medicine, Northwestern University, Chicago, IL, USA. ${ }^{5}$ Department of Radiology, Feinberg School of Medicine, Northwestern University, Chicago, IL, USA.

Received: 18 April 2018 Accepted: 15 May 2019

Published online: 21 June 2019

\section{References}

1. Schaefer O, Winterer J, Lohrmann C, Laubenberger J, Reichelt A, Langer M. Magnetic resonance imaging for supraspinatus muscle atrophy after cuff repair. Clin Orthop Relat Res. 2002;403:93-9.

2. Shen PH, Lien SB, Shen HC, Lee CH, Wu SS, Lin LC. Long-term functional outcomes after repair of rotator cuff tears correlated with atrophy of the supraspinatus muscles on magnetic resonance images. J Shoulder Elb Surg. 2008;17(1 Suppl):1S-7S.

3. Gladstone JN, Bishop JY, Lo IK, Flatow EL. Fatty infiltration and atrophy of the rotator cuff do not improve after rotator cuff repair and correlate with poor functional outcome. Am J Sports Med. 2007;35(5):719-28.

4. Rubino L, Stills HF Jr, Sprott DC, Crosby LA. Fatty infiltration of the torn rotator cuff worsens over time in a rabbit model. Arthroscopy. 2007;23(7):717-22.

5. Thomazeau H, Rolland Y, Lucas C, Duval JM, Langlais F. Atrophy of the supraspinatus belly. Assessment by MRI in 55 patients with rotator cuff pathology. Acta Orthop Scand. 1996;67(3):264-8.

6. Zanetti M, Gerber C, Hodler J. Quantitative assessment of the muscles of the rotator cuff with magnetic resonance imaging. Investig Radiol. 1998;33(3):163-70.

7. Goutallier D, Postel JM, Bernageau J, Lavau L, Voisin MC. Fatty muscle degeneration in cuff ruptures. Pre- and postoperative evaluation by CT scan. Clin Orthop Relat Res. 1994;(304):78-83.

8. Fuchs B, Weishaupt D, Zanetti M, Hodler J, Gerber C. Fatty degeneration of the muscles of the rotator cuff: assessment by computed tomography versus magnetic resonance imaging. J Shoulder Elb Surg. 1999:8(6):599-605.

9. Oh JH, Kim SH, Choi JA, Kim Y, Oh CH. Reliability of the grading system for fatty degeneration of rotator cuff muscles. Clin Orthop Relat Res. 2010; 468(6):1558-64

10. Slabaugh MA, Friel NA, Karas V, Romeo AA, Verma NN, Cole BJ. Interobserver and intraobserver reliability of the Goutallier classification using magnetic resonance imaging: proposal of a simplified classification system to increase reliability. Am J Sports Med. 2012;40(8):1728-34.

11. Dixon WT. Simple proton spectroscopic imaging. Radiology. 1984;153(1):189-94.

12. Crawford RJ, Volken T, Valentin S, Melloh M, Elliott JM. Rate of lumbar paravertebral muscle fat infiltration versus spinal degeneration in asymptomatic populations: an age-aggregated cross-sectional simulation study. Scoliosis Spinal Disord. 2016;11:21.

13. Elliott JM, Courtney DM, Rademaker A, Pinto D, Sterling MM, Parrish TB. The Rapid and Progressive Degeneration of the Cervical Multifidus in Whiplash: An MRI Study of Fatty Infiltration. Spine (Phila Pa 1976). 2015;40(12):E694-700.

14. Beattie KA, MacIntyre NJ, Ramadan K, Inglis D, Maly MR. Longitudinal changes in intermuscular fat volume and quadriceps muscle volume in the thighs of women with knee osteoarthritis. Arthritis Care Res (Hoboken). 2012;64(1):22-9.

15. Mankodi A, Bishop CA, Auh S, Newbould RD, Fischbeck KH, Janiczek RL Quantifying disease activity in fatty-infiltrated skeletal muscle by IDEAL- 
CPMG in Duchenne muscular dystrophy. Neuromuscul Disord. 2016;26(10): 650-8.

16. Nardo L, Karampinos DC, Lansdown DA, Carballido-Gamio J, Lee S, Maroldi $R$, et al. Quantitative assessment of fat infiltration in the rotator cuff muscles using water-fat MRI. J Magn Reson Imaging. 2014;39(5):1178-85.

17. Lee YH, Kim S, Lim D, Song HT, Suh JS. MR Quantification of the Fatty Fraction from T2*-corrected Dixon Fat/Water Separation Volumeinterpolated Breathhold Examination (VIBE) in the Assessment of Muscle Atrophy in Rotator Cuff Tears. Acad Radiol. 2015;22(7):909-17.

18. Smith AC, Parrish TB, Abbott R, Hoggarth MA, Mendoza K, Chen YF, et al. Muscle-fat MRI: 1.5 Tesla and 3.0 Tesla versus histology. Muscle Nerve. 2014; 50(2):170-6.

19. Matsumura N, Oguro S, Okuda S, Jinzaki M, Matsumoto M, Nakamura M, et al. Quantitative assessment of fatty infiltration and muscle volume of the rotator cuff muscles using 3-dimensional 2-point Dixon magnetic resonance imaging. J Shoulder Elb Surg. 2017.

20. Vidt ME, Santago AC 2nd, Tuohy CJ, Poehling GG, Freehill MT, Kraft RA, et al. Assessments of Fatty Infiltration and Muscle Atrophy From a Single Magnetic Resonance Image Slice Are Not Predictive of 3-Dimensional Measurements. Arthroscopy. 2016;32(1):128-39.

21. Walter SD, Eliasziw M, Donner A. Sample size and optimal designs for reliability studies. Stat Med. 1998;17(1):101-10.

22. Elliott JM, Walton DM, Rademaker A, Parrish TB. Quantification of cervical spine muscle fat: a comparison between T1-weighted and multi-echo gradient echo imaging using a variable projection algorithm (VARPRO). BMC Med Imaging. 2013:13:30

23. Fischer MA, Pfirrmann CW, Espinosa N, Raptis DA, Buck FM. Dixon-based MRI for assessment of muscle-fat content in phantoms, healthy volunteers and patients with achillodynia: comparison to visual assessment of calf muscle quality. Eur Radiol. 2014;24(6):1366-75.

24. Tingart MJ, Apreleva M, Lehtinen JT, Capell B, Palmer WE, Warner JJ. Magnetic resonance imaging in quantitative analysis of rotator cuff muscle volume. Clin Orthop Relat Res. 2003:415:104-10.

25. Juul-Kristensen B, Bojsen-Moller F, Finsen L, Eriksson J, Johansson G, Stahlberg F, et al. Muscle sizes and moment arms of rotator cuff muscles determined by magnetic resonance imaging. Cells Tissues Organs. 2000; 167(2-3):214-22.

26. Shrout P, Fleiss J. Intraclass correlations: Uses in assessing rater reliability. Psychol Bull. 1979;86:420-8.

27. Altman DG, Schulz KF, Moher D, Egger M, Davidoff F, Elbourne D, et al. The revised CONSORT statement for reporting randomized trials: explanation and elaboration. Ann Intern Med. 2001;134(8):663-94.

28. Stratford PW, Binkley FM, Riddle DL. Health status measures: strategies and analytic methods for assessing change scores. PhysTher. 1996;76(10):1109-23.

29. Stratford PW, Binkley J, Solomon P, Finch E, Gill C, Moreland J. Defining the minimum level of detectable change for the Roland-Morris questionnaire. Phys Ther. 1996;76(4):359-65.

30. Nozaki T, Tasaki A, Horiuchi S, Ochi J, Starkey J, Hara T, et al. Predicting Retear after Repair of Full-Thickness Rotator Cuff Tear: Two-Point Dixon MR Imaging Quantification of Fatty Muscle Degeneration-Initial Experience with 1-Year Follow-up. Radiology. 2016:151789.

31. Lehtinen JT, Tingart MJ, Apreleva M, Zurakowski D, Palmer W, Warner JJ. Practical assessment of rotator cuff muscle volumes using shoulder MRI. Acta Orthop Scand. 2003;74(6):722-9.

32. Valentin $\mathrm{S}$, Yeates TD, Licka T, Elliott J. Inter-rater reliability of trunk muscle morphometric analysis. J Back Musculoskelet Rehabil. 2015;28(1):181-90.

33. Lansdown DA, Lee S, Sam C, Krug R, Feeley BT, Ma CB. A Prospective, Quantitative Evaluation of Fatty Infiltration Before and After Rotator Cuff Repair. Orthop J Sports Med. 2017:5(7):2325967117718537.

34. Khoury V, Cardinal E, Brassard P. Atrophy and fatty infiltration of the supraspinatus muscle: sonography versus MRI. AJR Am J Roentgenol. 2008; 190(4):1105-11.

35. Warner JJ, Higgins L, IMt P, Dowdy P. Diagnosis and treatment of anterosuperior rotator cuff tears. J Shoulder Elb Surg. 2001;10(1):37-46.

\section{Ready to submit your research? Choose BMC and benefit from}

- fast, convenient online submission

- thorough peer review by experienced researchers in your field

- rapid publication on acceptance

- support for research data, including large and complex data types

- gold Open Access which fosters wider collaboration and increased citations

- maximum visibility for your research: over $100 \mathrm{M}$ website views per year

At $\mathrm{BMC}$, research is always in progress.

Learn more biomedcentral.com/submissions 